\title{
Efficacy of Ametoctradin 300 g/l + Dimethomorph 225 g/l SC on Downy Mildew Disease of Cucumber caused by Pseudoperonospora cubensis (Berk and Curt)
}

\author{
Ramesh", Vasant M. Ganiger and D. R. Patil
}

Main Horticulture Research and Extension Centre, University of Horticultural Sciences, Bagalkot- 587104, Karnataka, India

*Corresponding author

\section{A B S T R A C T}

\begin{tabular}{|l|}
\hline Key w o r d s \\
$\begin{array}{l}\text { Pseudoperonospora } \\
\text { cubensis, Downy } \\
\text { Mildew disease, } \\
\text { Cucumber }\end{array}$ \\
\hline Article Info \\
\hline $\begin{array}{l}\text { Accepted: } \\
\text { 24 July 2020 } \\
\text { Available Online: } \\
\text { 10 August } 2020\end{array}$ \\
\hline
\end{tabular}

\begin{abstract}
Cucumber (Cucumis sativus) is a widely cultivated crop in the gourd family and plays an important role in our daily diet. However, the cucumber often suffers from disease during reproductive stage that affects the output and quality of cucumber. The commercial cultivars are highly susceptible to downy mildew disease which causes huge losses both in fruit quality and yield. During Rabi-2015-16 and Kharif- 2016-17, field study were conducted at Main Horticultural Research and Extension Centre, Bagalkot on the management of downy mildew disease by using different concentrations of Ametoctradin 300g/l + Dimethomorph 225g/l SC, Metalaxyl 8\% + Mancozeb 64\% WP, Dimethomorph 50\%WP and Cymoxanil 8\% + Mancozeb 64\% WP. Among these treatments, Ametoctradin 300g/l+ Dimethomorph 225g/l SC @ 1000ml/ha was recorded least PDI and highest yield as compare to other treatments. It was followed by Metalaxyl 8\%+ Mancozeb $64 \% \mathrm{WP}$ at $1500 \mathrm{~g} / \mathrm{ha}$, Cymoxanil $8 \%+$ Mancozeb $64 \% \mathrm{WP}$ at $1500 \mathrm{~g} / \mathrm{ha}$ and Ametoctradin 300g/l + Dimethomorph 225g/l SC @ 800ml/ha.
\end{abstract}

\section{Introduction}

Cucumber (Cucumis sativus L.) is of Asiatic origin (De Candolle 1883) and The cultivation of crop goes back to at least 3000 years in India and 2000 years in China (Robinson and Decker-Walters, 1997). In India, a number of major and minor cucurbits are cultivated, which share about 5.6 per cent of the total vegetable production (Rai, et al., 2008). The genus Cucumis contains 52 species, of which Cucumis sativus and Cucumis melo are the most economically important species grown as vegetables (Ghebretinsae et al., 2007). They supply carbohydrates, proteins, fats, vitamins, and mineral elements which are the most essential requirements of our body. In India, cucumber is grown in an area of 0.82 lakh hectares with a production of 12.60 lakh tones and productivity of 15.37 tones/ha (NHB, 2018). The prominent cucumber growing states are Haryana, Madhya Pradesh, Karnataka, Andhra Pradesh, Uttar Pradesh and Punjab. In Karnataka, it occupies an area of 8.27 thousand hectares with the production of 
131.96 thousand tones and productivity of 15.96 tones/ha. Bellary, Haveri, Mandya and Hassan are major districts of cucumber cultivation (NHB, 2018).

Downy mildew caused by fungi Pseudoperonospora cubensis (Berk and Curt) is one of the most destructive diseases. Symptoms firstly appear as pale green areas on the upper leaf surfaces and later change to yellow angular spots. A fine dark brown to grayish downy growth soon appears on the lower leaf surface. Infected leaves generally die but may remain erect while the edges of the leaf blades curl inward. The present fungicides available in market against downy mildew disease are becoming less effective in controlling the disease. There is need to evaluate the efficacy of new combi product against downy mildew disease of cucumber. In this regard a combi product Ametoctradin $300 \mathrm{~g} / \mathrm{l}+$ Dimethomorph 225g/l SC was evaluated at different concentration against the downy mildew of cucumber during Rabi2015-16 and Kharif-2016-17 at Main Horticulture Research and Extension Centre, Univeristy of Horticultural Sciences, Bagalkot.

\section{Materials and Methods}

An experiment was conducted at Main Horticulture Research and Extension Centre, Bagalkot during Rabi 2015-16 and Kharif 2016-17 to study the efficacy and phytotoxicity of combi product ametoctradin $300 \mathrm{~g} / 1$ + dimethomorph225 g/l SC against downy mildew disease of cucumber. The technical grade of ametoctradin $300 \mathrm{~g} / \mathrm{l}+$ dimethomorph225 g/l SC was supplied by BASF India Limited, Mumbai and remaining fungicides were purchased from local pesticide shop. It was conducted in randomized block design with seven treatments and three replications. Seeds of cucumber hybrid $F_{1}$ (Sakata) were sowed in the plot $(4.5 \mathrm{~m} \times 7.5 \mathrm{~m})$ with a spacing of 1.5 $\mathrm{m} \times 0.75 \mathrm{~m}$. the treatments were imposed while once the disease appearance stage (DAS) of downy mildew i.e. 32 days after sowing. The sprays were repeated at ten days interval 500-750 liters of water per hector and two sprays were given at 10 days interval. The observations were recorded before first spray and ten days after each sprays. The severity of the disease was measured using 09 disease rating scale given by Wheeler (1969).

\begin{tabular}{|c|l|}
\hline Grades & \multicolumn{1}{|c|}{ Symptoms } \\
\hline 0 & No disease symptoms (Nil) \\
\hline 1 & $\begin{array}{l}1-5 \text { per cent of leaf area covered/ } \\
\text { infected }\end{array}$ \\
\hline 3 & $\begin{array}{l}6-10 \text { per cent of leaf area covered/ } \\
\text { infected }\end{array}$ \\
\hline 5 & $\begin{array}{l}11-25 \text { per cent of leaf area } \\
\text { covered/ infected }\end{array}$ \\
\hline 7 & $\begin{array}{l}\text { 26-50 per cent of leaf area } \\
\text { covered/ infected }\end{array}$ \\
\hline 9 & $\begin{array}{l}>50 \text { per cent of leaf area covered/ } \\
\text { infected }\end{array}$ \\
\hline
\end{tabular}

Per cent disease index (PDI) was calculated by using the formula given by Wheeler (1969)

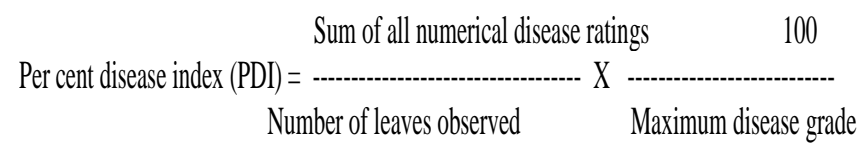

\section{Results and Discussion}

The two season data on efficacy of ametoctradin $300 \mathrm{~g} / 1$ + dimethomorph $225 \mathrm{~g} / 1$ $\mathrm{SC}$ on the downy mildew disease and yield of cucumber presented in tables 1 and 2 respectively.

First season (2015-16)

\section{Downy mildew incidence}

After second spray, the per cent disease index was 15 per cent in the plot sprayed with 
ametoctradin $300 \mathrm{~g} / 1+$ dimethomorph $225 \mathrm{~g} / 1$ $\mathrm{SC} @ 1000 \mathrm{ml} / \mathrm{ha}$ which was minimum among all treatments and significantly on par to next best treatment metalaxyl 8\%+ mancozeb 64\% WP @ 1500g/ha (16.20 PDI), ametoctradin $300 \mathrm{~g} / 1+$ dimethomorph $225 \mathrm{~g} / \mathrm{l}$ SC @ $800 \mathrm{ml} / \mathrm{ha}$ and cymoxanil 8\% + mancozeb 64\% WP @ 1500g/ha (17.73 PDI).

However, ametoctradin $300 \mathrm{~g} / \mathrm{l}+$ dimethomorph 225 g/l SC @ 1000 ml/ha was found to be significantly superior to ametoctradin $300 \mathrm{~g} / 1+$ dimethomorph $225 \mathrm{~g} / 1$ SC @ 600 ml/ha and dimethomorph 50\% WP ( 1000g/ha (21.40 PDI).

Untreated control recorded highest per cent disease incidence (35.83 PDI). Downy mildew disease control was achieved by $58.14,54.79$ and 52.00 per cent in plot sprayed with ametoctradin $300 \mathrm{~g} / \mathrm{l}+$ dimethomorph $225 \mathrm{~g} / \mathrm{l} \mathrm{SC} @ 1000 \mathrm{ml} / \mathrm{ha}$, metalaxyl 8\%+ mancozeb 64\% WP @ $1500 \mathrm{~g} / \mathrm{ha}$ and ametoctradin $300 \mathrm{~g} / \mathrm{l}+$ dimethomorph $225 \mathrm{~g} / \mathrm{l} \mathrm{SC}$ @ $800 \mathrm{ml} / \mathrm{ha}$ respectively over untreated control (Table 1).

\section{Fruit yield}

Infection of downy mildew disease was not noticed in fruits. However, it reduced the plant photosynthesis activity as a result overall fruit yield was reduced.

The treatment ametoctradin $300 \mathrm{~g} / \mathrm{l}+$ dimethomorph 225 g/l SC @ 1000 ml/ha was recorded highest fruit yield (23.33 q/ha) and was significantly superior to standard checks metalaxyl 8\%+ mancozeb 64\% WP @ $1500 \mathrm{~g} / \mathrm{ha}(21.92 \mathrm{q} / \mathrm{ha})$ and cymoxanil $8 \%+$ mancozeb 64\% WP @ 1500g/ha (21.00 q/ha).

The untreated control recorded significantly least fruit yield $(12.03 \mathrm{q} / \mathrm{ha})$ than all other treatments (Table 1).

\section{Second season (2016-17)}

\section{Downy mildew incidence}

During second season also same trend was followed that the severity of downy mildew disease differed significantly with respect to all treatments at all stages of observation. Downy mildew disease incidence was significantly least in ametoctradin $300 \mathrm{~g} / \mathrm{l}+$ dimethomorph $225 \mathrm{~g} / \mathrm{l}$ SC @ $1000 \mathrm{ml} / \mathrm{ha}$ (32.89 PDI) and which was significantly on par with cymoxanil $8 \%$ + mancozeb $64 \%$ WP @ 1500g/ha (39.41 PDI) and metalaxyl 8\%+ mancozeb 64\% WP @ 1500g/ha (39.70 PDI).

However, the treatment ametoctradin $300 \mathrm{~g} / \mathrm{l}$ + dimethomorph 225 g/l SC @ 1000 ml/ha was significantly superior over ametoctradin $300 \mathrm{~g} / 1$ + dimethomorph $225 \mathrm{~g} / 1 \mathrm{SC} @ 800$ $\mathrm{ml} / \mathrm{ha}$ (42.81 PDI), ametoctradin $300 \mathrm{~g} / \mathrm{l}+$ dimethomorph $225 \mathrm{~g} / \mathrm{l} \quad \mathrm{SC}$ @ $600 \mathrm{ml} / \mathrm{ha}$ (45.19 PDI) and dimethomorph 50\% WP @ $1000 \mathrm{~g} / \mathrm{ha}$ (50.96 PDI). The untreated control (59.26 PDI) recorded significantly highest percent disease index (Table 2).

\section{Fruit yield}

The treatments differed significantly with respect to fruit yield of cucumber. Ametoctradin $300 \mathrm{~g} / 1$ + dimethomorph $225 \mathrm{~g} / 1$ SC @ 1000 ml/ha (24.23 q/ha) recorded significantly highest yield than all other treatments and significantly on par with the metalaxyl 8\%+ mancozeb 64\% WP @ $1500 \mathrm{~g} / \mathrm{ha} \quad(23.20 \mathrm{q} / \mathrm{ha})$. However, the treatment ametoctradin $300 \mathrm{~g} / \mathrm{l}+$ dimethomorph 225 g/l SC @ 1000 ml/ha was significantly superior over standard check cymoxanil 8\% + mancozeb 64\% WP @ $1500 \mathrm{~g} / \mathrm{ha}(21.60 \mathrm{q} / \mathrm{ha})$ and dimethomorph $50 \%$ WP @ 1000g/ha (12.33 q/ha). The untreated control $(9.50 \mathrm{q} / \mathrm{ha})$ recorded least yield (Table 2). 
Table.1 Effect of Ametoctradin 300 g/l + Dimethomorph 225 g/l SC on incidence of downy mildew of cucumber during 2015-16

\begin{tabular}{|c|c|c|c|c|c|c|c|}
\hline \multirow[t]{2}{*}{ Tr.No. } & \multirow[t]{2}{*}{ Treatments } & \multirow{2}{*}{$\begin{array}{l}\text { Formulation } \\
\text { (ml or g/ha) }\end{array}$} & \multicolumn{3}{|c|}{ Per cent disease index (PDI) of downy } & \multirow{2}{*}{$\begin{array}{l}\text { Per cent } \\
\text { disease } \\
\text { reduction } \\
\text { over } \\
\text { control }\end{array}$} & \multirow[t]{2}{*}{ Yield (q/ha) } \\
\hline & & & $\begin{array}{l}\text { Before } \\
\text { Spray }\end{array}$ & $\begin{array}{l}10 \text { days after } \\
1^{\text {st }} \text { spray }\end{array}$ & $\begin{array}{l}10 \text { days after } \\
2^{\text {nd }} \text { spray }\end{array}$ & & \\
\hline 1 & $\begin{array}{l}\text { Ametoctradin } 300 \mathrm{~g} / 1+ \\
\text { Dimethomorph } 225 \mathrm{~g} / 1 \mathrm{SC}\end{array}$ & 800 & $\begin{array}{c}7.67 \\
(16.07)^{*}\end{array}$ & $\begin{array}{c}15.53 \\
(23.21)\end{array}$ & $\begin{array}{c}17.20 \\
(24.46)\end{array}$ & 52.00 & 21.27 \\
\hline 2 & $\begin{array}{l}\text { Ametoctradin } 300 \mathrm{~g} / 1+ \\
\text { Dimethomorph } 225 \mathrm{~g} / \mathrm{l} \mathrm{SC}\end{array}$ & 1000 & $\begin{array}{c}7.60 \\
(16.00)\end{array}$ & $\begin{array}{c}12.67 \\
(20.81)\end{array}$ & $\begin{array}{c}15.00 \\
(24.32)\end{array}$ & 58.14 & 23.33 \\
\hline 3 & $\begin{array}{l}\text { Ametoctradin } 300 \mathrm{~g} / 1+ \\
\text { Dimethomorph } 225 \mathrm{~g} / 1 \mathrm{SC}\end{array}$ & 600 & $\begin{array}{c}7.50 \\
(15.89)\end{array}$ & $\begin{array}{c}15.47 \\
(23.16)\end{array}$ & $\begin{array}{c}20.40 \\
(26.75)\end{array}$ & 43.06 & 20.02 \\
\hline 4 & $\begin{array}{l}\text { Metalaxyl 8\%+ Mancozeb } \\
64 \% \text { WP }\end{array}$ & 1500 & $\begin{array}{c}7.47 \\
(15.85)\end{array}$ & $\begin{array}{c}13.60 \\
(21.60)\end{array}$ & $\begin{array}{c}16.20 \\
(23.70)\end{array}$ & 54.79 & 21.92 \\
\hline 5 & Dimethomorph 50\% WP & 1000 & $\begin{array}{c}7.33 \\
(15.71)\end{array}$ & $\begin{array}{c}15.20 \\
(22.93)\end{array}$ & $\begin{array}{c}21.40 \\
(27.52)\end{array}$ & 40.27 & 19.51 \\
\hline 6 & $\begin{array}{l}\text { Cymoxanil 8\% + } \\
\text { Mancozeb 64\% WP }\end{array}$ & 1500 & $\begin{array}{c}7.23 \\
(15.60)\end{array}$ & $\begin{array}{c}14.30 \\
(22.78)\end{array}$ & $\begin{array}{c}17.73 \\
(24.90)\end{array}$ & 50.52 & 21.00 \\
\hline 7 & Untreated control & -- & $\begin{array}{c}7.63 \\
(16.04)\end{array}$ & $\begin{array}{c}28.33 \\
(32.13)\end{array}$ & $\begin{array}{c}35.83 \\
(40.54)\end{array}$ & --- & 12.03 \\
\hline & & SEm \pm & 0.19 & 0.78 & 1.20 & & 9.13 \\
\hline & & CD@5\% & 0.55 & 2.28 & 3.53 & & 0.16 \\
\hline & & CV\% & 4.36 & 8.12 & 10.72 & & 0.51 \\
\hline
\end{tabular}

*figures on parenthesis are angular transformed values 
Table.2 Effect of Ametoctradin $300 \mathrm{~g} / 1$ + Dimethomorph $225 \mathrm{~g} / 1 \mathrm{SC}$ on incidence of downy mildew of cucumber during Kharif- 2016-17

\begin{tabular}{|c|c|c|c|c|c|c|c|}
\hline \multirow[t]{2}{*}{ Tr.No. } & \multirow[t]{2}{*}{ Treatments } & \multirow{2}{*}{$\begin{array}{l}\text { Formulation } \\
\text { (ml or g/ha) }\end{array}$} & \multicolumn{3}{|c|}{ Per cent disease index (PDI) of downy } & \multirow{2}{*}{$\begin{array}{l}\text { Per cent disease } \\
\text { reduction over } \\
\text { control }\end{array}$} & \multirow{2}{*}{$\begin{array}{l}\text { Yield } \\
\text { (q/ha) }\end{array}$} \\
\hline & & & $\begin{array}{l}\text { Before } \\
\text { Spray }\end{array}$ & $\begin{array}{l}10 \text { days after } \\
1^{\text {st }} \text { spray }\end{array}$ & $\begin{array}{l}10 \text { days after } \\
2^{\text {nd }} \text { spray }\end{array}$ & & \\
\hline 1 & $\begin{array}{l}\text { Ametoctradin } 300 \mathrm{~g} / \mathrm{l}+ \\
\text { Dimethomorph } 225 \mathrm{~g} / \mathrm{S} \mathrm{SC}\end{array}$ & 800 & $\begin{array}{c}3.70 \\
(11.09)^{*}\end{array}$ & $\begin{array}{c}44.00 \\
(26.42)\end{array}$ & $\begin{array}{c}42.81 \\
(26.03)\end{array}$ & 23.74 & 20.05 \\
\hline 2 & $\begin{array}{l}\text { Ametoctradin } 300 \mathrm{~g} / 1+ \\
\text { Dimethomorph } 225 \mathrm{~g} / \mathrm{l} \mathrm{SC}\end{array}$ & 1000 & $\begin{array}{c}3.50 \\
(10.78)\end{array}$ & $\begin{array}{c}32.89 \\
(22.61)\end{array}$ & $\begin{array}{c}36.74 \\
(23.96)\end{array}$ & 38.00 & 24.23 \\
\hline 3 & $\begin{array}{l}\text { Ametoctradin } 300 \mathrm{~g} / \mathrm{l}+ \\
\text { Dimethomorph } 225 \mathrm{~g} / \mathrm{S} \mathrm{SC}\end{array}$ & 600 & $\begin{array}{c}3.63 \\
(10.98)\end{array}$ & $\begin{array}{c}44.30 \\
(26.51)\end{array}$ & $\begin{array}{c}45.19 \\
(26.80)\end{array}$ & 27.76 & 19.30 \\
\hline 4 & $\begin{array}{l}\text { Metalaxyl 8\%+ Mancozeb } \\
64 \% \text { WP }\end{array}$ & 1500 & $\begin{array}{c}3.65 \\
(11.20)\end{array}$ & $\begin{array}{c}35.63 \\
(23.60)\end{array}$ & $\begin{array}{c}39.70 \\
(24.96)\end{array}$ & 33.01 & 23.20 \\
\hline 5 & Dimethomorph 50\% WP & 1000 & $\begin{array}{c}3.59 \\
(10.93)\end{array}$ & $\begin{array}{c}50.59 \\
(28.45)\end{array}$ & $\begin{array}{c}50.96 \\
(28.60)\end{array}$ & 14.01 & 12.33 \\
\hline 6 & $\begin{array}{l}\text { Cymoxanil 8\% + } \\
\text { Mancozeb 64\% WP }\end{array}$ & 1500 & $\begin{array}{c}3.60 \\
(10.94)\end{array}$ & $\begin{array}{c}36.74 \\
(23.98)\end{array}$ & $\begin{array}{c}39.41 \\
(24.88)\end{array}$ & 33.50 & 21.60 \\
\hline 7 & Untreated control & -- & $\begin{array}{c}3.70 \\
(11.09)\end{array}$ & $\begin{array}{c}52.74 \\
(29.15)\end{array}$ & $\begin{array}{c}59.26 \\
(31.08)\end{array}$ & --- & 9.50 \\
\hline & & SEm \pm & 0.20 & 1.70 & 1.83 & & 0.38 \\
\hline & & CD@5\% & 0.65 & 4.99 & 5.38 & & 1.17 \\
\hline & & $\mathrm{CV} \%$ & 3.92 & 6.93 & 7.07 & & 4.42 \\
\hline
\end{tabular}

*figures on parenthesis are angular transformed values 
From the two year study (Rabi 2015-16 \& Kharif 2016-17), it was revealed that the new combi product ametoctradin $300 \mathrm{~g} / \mathrm{l}+$ dimethomorph $225 \mathrm{~g} / \mathrm{l} \mathrm{SC} @ 800 \mathrm{ml} / \mathrm{ha}$ or $1000 \mathrm{ml} / \mathrm{ha}$ were quite effective for management of downy mildew disease of cucumber and recorded higher fruit yield than rest of the treatments. Rajesh kumar (2013), reported that metalaxyl + mancozeb (Ridomil MZ \& Ridomil gold) at 0.25 per cent concentration having more persistence on cucumber leaf that other fungicides tested. The different commercial form of metalaxyl + mancozeb (Ridomil MZ \& Ridomil gold) and cymoxanil + mancozeb (Curzate M-8) at 0.25 per cent concentration recorded lowest disease incidence and highest fruit yield (Javid Ahmad Bhat et al., 2018). Khan (1999) reported that, alternative spray of Ridomil MZ- 72 (Metalaxyl + Mancozeb) and Dithane M-45 (Mancozeb), gave good control of downy mildew of cucumber, highest net benefit and increase in the yield considerably in comparison with the other treatments.

\section{References}

De Candolle, A. C. P. (1883). Origine des plantes cultivées. Pp. 210-12. Germer Baillière et Cie, Paris.

Ghebretinsae, A, G., Thulin, M. and Barber, J. C. (2007). Nomenclature changes in Cucumis (Cucurbitaceae). Novon., 17:176-78.
Javid Ahmad Bhat., Rizwan Rashid., Waseem Ali Dar and R. A. Bhat. (2018). Efficacy of different fungicides for the management of downy mildew of cucumber grown under low plastic tunnel. International journal of pure and applied bioscience., 6(2): 884-890.

Khan, A. R. (1999). Evaluation of fungicides and time of application for controlling downy mildew of cucumber. Pak J. Phytopath 11: 169-72.

National Horticultural Board. (2018). Horticultural statistics at a glance. Ministry of Agriculture Farmers Welfare, Government of India.

Rai, M., Pandey, S. and Kumar, S. (2008). Cucurbit research in India: a retrospect. Proceedings of the IXth EUCARPIA meeting on genetics and breeding of Cucurbitaceae (Pitrat M, ed), Pp 28593 INRA, Avignon (France).

Rajesh Kumar. (2013). Persistence of fungicides on cucumber (Cucumis sativus L.) in relation to downy mildew disease. M. Sc. (Agri.) Thesis, Punjab Agricultural University Ludhiana.

Robinson, R. W. and Decker-Walters, D. S. (1997). Cucurbits. Pp 1-22. CAB International, New York.

Wheeler, B.E.J., 1969, An Introduction to Plant Diseases, John Wiley and Sons Ltd., London, p. 301

\section{How to cite this article:}

Ramesh, Vasant M. Ganiger and Patil, D. R. 2020. Efficacy of Ametoctradin 300 g/l + Dimethomorph $225 \mathrm{~g} / 1 \mathrm{SC}$ on Downy Mildew Disease of Cucumber caused by Pseudoperonospora cubensis (Berk and Curt). Int.J.Curr.Microbiol.App.Sci. 9(08): 29792984. doi: https://doi.org/10.20546/ijcmas.2020.908.335 